\title{
"Smart inhibition": electrophysiological evidence for the suppression of conflict-generating task rules during task switching
}

\author{
Nachshon Meiran • Shulan Hsieh • Chi-Chih Chang
}

Published online: 18 May 2011

(C) Psychonomic Society, Inc. 2011

\begin{abstract}
A major challenge for task switching is maintaining a balance between high task readiness and effectively ignoring irrelevant task rules. This calls for finely tuned inhibition that targets only the source of interference without adversely influencing other task-related representations. The authors show that irrelevant task rules generating response conflict are inhibited, causing their inefficient execution on the next trial (indicating the presence of competitor rule suppression[CRS];Meiran, Hsieh, \& Dimov, Journal of Experimental Psychology: Learning, Memory and Cognition, 36, 992-1002, 2010). To determine whether CRS influences task rules, rather than target stimuli or responses, the authors focused on the processing of the task cue before the target stimulus was presented and before the response could be chosen. As was predicted, CRS was found in the event-related potentials in two time windows during task cue processing. It was also found in three time windows after target presentation. Source localization analyses suggest the involvement of the right dorsal prefrontal cortex in all five time windows.
\end{abstract}

\author{
N. Meiran $(\bowtie)$ \\ Department of Psychology and Zlotowsky Center for \\ Neuroscience, Ben-Gurion University of the Negev, \\ Beer-Sheva, Israel 84105 \\ e-mail: nmeiran@bgu.ac.il \\ S. Hsieh $(\bowtie)$ \\ National Cheng Kung University, \\ Tainan, Taiwan \\ e-mail: psyhs1@mail.ncku.edu.tw \\ C.-C. Chang \\ National Chung Cheng University, \\ Chia-Yi, Taiwan
}

Keywords Event-related potentials · Reaction time · Task switching $\cdot$ Cognitive control $\cdot$ Inhibition $\cdot$ Conflict monitoring

\section{Introduction}

A key characteristic of human cognitive control is its remarkable flexibility. Humans can contextualize their learned skills, expressing a given skill only in its appropriate context and avoiding skills that are inappropriate for that context. The cognitive and neuronal mechanisms that enable such contextual flexibility have been a target of intense research efforts over the last 15 years or so. Perhaps the most popular paradigm in this research is the taskswitching paradigm (see Karayanidis et al., 2010; Kiesel et al., 2010; Meiran, 2010; Monsell, 2003; Vandierendonck, Liefooghe, \& Verbruggen, 2010, for a review; see Koch, Gade, Schuch, \& Philipp, 2010, for a review on inhibition in task switching), in which the task context frequently changes.

In a typical task-switching paradigm, participants are asked to classify multidimensional objects according to a particular dimension. For example, in the experiment reported below, participants had to classify target stimuli according to shape, color, or two location dimensions (in vs. out and up vs. down; see Fig. 1). In this paradigm, the relevant dimension for classification changed on every trial, as instructed by a transparent task cue. Below, we refer to each dimension (e.g., shape) and the rules relating values along this dimension (e.g., dot and line) to responses (e.g., right key, left key) as a task rule. 
Fig. 1 The behavioral paradigm. a Schematic representation of the events in a trial, including cue presentation, followed by target presentation; b the four target objects; c The cues for the four tasks

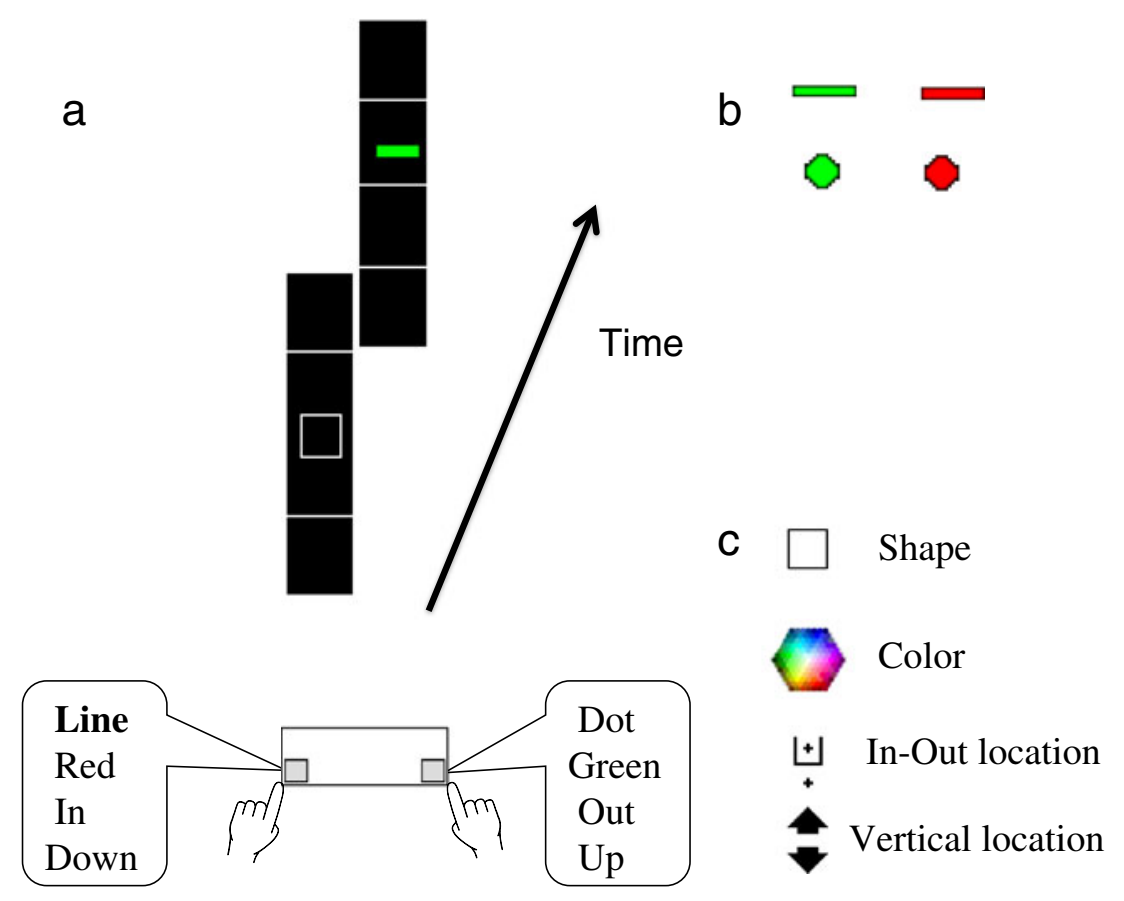

Most of the studies that have used the task-switching paradigm have focused on the costs associated with task switching. Unlike these studies, the present work focuses on a control dilemma that is invoked in situations involving frequent task switches.

\section{Control dilemma in task switching}

As was noted by Goschke (2000), cognitive control often involves maintaining a delicate balance between conflicting demands. In task switching, the conflict arises because participants need to maintain high task readiness while remaining focused on the relevant task rule and ignoring the other (currently irrelevant) task rules. We argue that maintaining high task readiness and focusing are conflicting demands and refer to them as readiness and focusing, as elaborated below.

Readiness Because of the frequent and unpredictable task switches, participants need to be constantly ready to execute any given task among the tasks that are involved in the given experimental block, and they do so by holding the task rules in a highly accessible state. Moreover, because all of the rules (including the rules that are currently irrelevant for the specific trial) are highly accessible, these rules may easily get activated, leading to an inappropriate response. Such an inappropriate response activation is reflectedin the task rule congruency effect (TRCE; see Meiran \& Kessler, 2008, for a review). The TRCE is demonstrated by the relatively poor performance on incongruent trials, relative to congruent trials. On incongruent trials, the irrelevant rules activate competing responses (e.g., the relevant rule indicates key 1 as the correct response, while an irrelevant rule indicates key 2 as the correct response). On congruent trials, there is no such response conflict, since the relevant rule and the irrelevant rule activate the same response. This performance difference indicates the fact that the currently irrelevant rules are not completely shut off.

Focusing Because of the need to maintain high readiness, responses generated by currently irrelevant rules become activated and may erroneously get executed. To prevent that from happening, participants need to focus on the relevant rule and/or inhibit the incorrect rules. The question is then how such focusing is achieved.

Experiments that did not involve task switching suggested several focusing mechanisms, but these mechanisms are not always sufficiently fine tuned and, therefore, may violate the delicate balance between readiness and focusing, as explained below. For example, according to conflict-monitoring theory (Botvinick, Braver, Carter, Barch, \& Cohen, 2001), the anterior cingulate cortex quantifies the degree of conflict and sends control signals that handle the source of conflict. An important characteristic of this theory (as currently formulated) is that the system reacts to the conflict by activating task-relevant representations (Brown, Reynolds, \& Braver, 2007; Egner \& Hirsch, 2005). The problem is that activating the relevant rule likely makes it difficult to switch away from this rule on the next trial (see Allport, Styles, \& Hsieh, 1994; Yeung \& Monsell, 2003; Yeung, Nystrom, Aronson, \& Cohen, 2006). Alternatively, conflict monitoring 
could operate through the inhibition of all of the currently irrelevant rules once a conflict has been detected. Yet such indiscriminate rule inhibition might make it difficult to switch tasks on the next trial when one of the inhibited rules becomes relevant. Finally, the competing response (e.g., pressing key 2) may be inhibited, but this strategy runs the risk that the inhibited response will be required on the next trial.

In contrast to these indiscriminate mechanisms, there are more finely targeted focusing mechanisms that impair readiness only minimally (see, e.g., Tipper, 2001, for a review). In a recent behavioral experiment (Meiran, Hsieh, \& Dimov, 2010), we showed evidence for a finely targeted mechanism that operates only on the rule that has generated the response conflict and does so at the relatively abstract level of the task rule. We labeled it competitor rule suppression (CRS). The goal of the present study was to test Meiran et al.'s claim that CRS influences abstract rules, as opposed to more concrete task representations, such as a specific interpretation of the stimulus (e.g., red; see Tipper, 2001) or a specific manual response.

To demonstrate CRS, Meiran et al. (2010) used a paradigm involving four tasks: two location tasks (up-down, right-left) and two tasks performed on photographs of faces (gender and hair color). Thus, each trial involved a relevant (to-beexecuted) task rule and three irrelevant task rules, some of which could activate a competing response. In our paradigm, the tasks always switched, but aside from that constraint, they were pseudorandomly ordered. (Note that the fact that all the trials involved a task switch prevented us from measuring switch costs, because the measurement of switch costs requires a comparison between task switch trials and task repetition trials, but the latter were absent in our design.)

Meiran et al.'s (2010) critical finding concerns the fate of the task rules that have generated a response conflict (henceforth, competing rules). This fate was examined by comparing specific scenarios. All of these scenarios defined by the relationship between the competing rule on trial $n-1$ and the relevant task rule on trial $n$ (for which performance and event-related potentials [ERPs] were analyzed). As will become clear immediately, the scenarios are defined according to the type of transition between trial $n-1$ and trial $n$ (see Table 1 for concrete examples). Here, we focus on three scenarios.

$C R S+$ In this scenario, the competing task rule from trial $n-1$ became the relevant rule on trial $n$. We reasoned that if the competing rule has been suppressed on trial $n-1$, its execution on trial $n$ would be impaired, relative to a relevant baseline, as was found by Meiran et al. (2010).

Post-incongruent In this scenario, there was a response conflict on trial $n-1$, but the relevant task rule on trial $n$ was not the competing rule from trial $n-1$.
Table 1 Examples for the conditions involved in the critical comparisons

\begin{tabular}{ll}
\hline Condition & Example \\
\hline Congruency Effect & $\begin{array}{l}\text { Stimulus on Trial } n^{*} \\
\text { Upper-inner red line }\end{array}$ \\
Congruent & Upper-inner red dot \\
1 competing rule & $\underline{\text { Lower-inner red } \mathbf{\text { dot }}}$ \\
2 competing rules & $\underline{\text { Lower-outer red }}$ \\
3 competing rules & \\
CRS and Conflict Adaptation Effects & \\
$\begin{array}{l}\text { Post-congruent (no rule is suppressed) } \\
\text { CRS + (relevant rule is shape and } \\
\text { suppressed rule is shape) }\end{array}$ & $\begin{array}{l}\text { Upper-inner red line } \\
\text { Post-incongruent (relevant rule is shape } \\
\text { and suppressed rule is up-down) }\end{array}$ \\
\hline
\end{tabular}

Note. The examples refer to the following response mapping:key 1 , "red," "line," "up,", "in"; key 2, "green," "dot," "down," "out"

*Relevant rule is color. Correct response is key 1 (because red is relevant and mapped to key 1). Stimulus values that go with key 2 (and hence, produce a response conflict) are highlighted with boldface and underlining.

**Relevant rule on trial $n-1$ is color and on trial $n$ is shape. Stimulus values that were associated with a conflict generating task rules on trial $n-1$ are highlighted in boldface and underline.

Post-congruent In this scenario, there was no response conflict on trial $n-1$.

The three scenarios were compared by employing two theoretically driven orthogonal planned contrasts. The $C R S$ contrast was between CRS+ trials and post-incongruent trials. This contrast reflects the lingering inhibition of the competing rule from trial $n-1$. Given the fact that we were comparing three conditions, there were two orthogonal contrasts describing their variance. We therefore decided to also examine the additional contrast that describes the remaining variance among the three conditions. This contrast (which is orthogonal to the CRS contrast) compares the average of two conditions, post-incongruent and CRS+, with post-congruent. We label this contrast conflict adaptation. We wish to comment here that our design was optimally suited to examine the CRS contrast but was less well suited to study the conflict adaptation contrast, as is explained in the General Discussion section.

Research question and predictions

The present work addressed the core question: Does CRS influence task rules (rather than only more concrete representations)? In this regard, Meiran et al. (2010) measured reaction time (RT) and accuracy following target presentation on trial $n$. Because of this feature of their 
design, it remains possible that CRS influenced only stimulusrelated representations or response-specific processes.

One way to show that CRS operates (also) at the level of the task rule is to examine how it influences ERPs during the cue epoch, capitalizing on the fact that the task cue preceded the target stimulus. Note that the task cue indicated the identity of the next task (such as shape), but it specified neither the nature of the next target stimulus (e.g., a red dot presented in the outer and upper segment of the display), nor the next response (e.g., key 1), nor the nature of the stimulus-response rule that was invoked (e.g., "IF \{dot\} THEN \{Press Key 1\}"). Because the period devoted to cue processing is when task identities or task rules are retrieved, among other things (Mayr \& Kliegl, 2000, 2003), an influence of CRS on ERP during this epoch would indicate that CRS operates on task rules. Thus, our goal was to test whether CRS influences cuelocked ERPs in the cue period, before the target stimulus has been presented. Note the fact that all the trials in our experiment involved task switching. This implies that there was no need to deal with the potential confound between task repetition and (task) cue repetition. ${ }^{1}$

Although our focus was on the cue epoch as examined in cue-locked ERPs, we also analyzed the ERPs in the response-to-cue epoch and the target epoch, for completeness sake. In the next section, we briefly review the relevant literature on task switching and make predictions regarding each one of these three epochs. The reader who wishes to focus just on the main issue may skip the sections on response epoch and target epoch.

Response epoch In this analysis, we focused on the epoch following the $n-1$ response and lasting until the $n$th cue. The response epoch is of special interest for two reasons. One reason is more technical, and the other is theoretical. The technical motivation concerns the validation of our analytic approach. Specifically, the CRS+ and post-incongruent conditions are not differentiated conceptually until the $n$th cue is presented, because it is the relationship between the incongruence on trial $n-1$ and the task on trial $n$ that determine the difference between these two conditions. Thus, the fact that the CRS contrast was not significant during the response epoch would show that our statistical procedure was sufficiently conservative.

The theoretical motivation concerns the conflictmonitoring theory (Botvinick et al., 2001), which might

\footnotetext{
${ }^{1}$ Specifically, in studies involving task repetitions, when the task repeats so does the task cue, thus creating a confound (Logan \& Bundesen, 2003; Mayr \& Kliegl, 2003; see also Forstmann, Brass, \& Koch, 2007; Garcia-Garcia, Barceló, Clemente, \& Escera, 2010; Jost, Mayr, \& Rösler, 2008; Lorist et al., 2000; Periáñez \& Barceló, 2009; West, Langley, \& Bailey, 2010).
}

suggest that the adaptation that follows detected conflicts could take place during the response epoch. However, authors who have developed this theory have suggested that conflict monitoring takes place before the response in the case of correct trials but takes place during or after the response in the case of errors, because the conflict between the erroneous and the correct response can take place only once the response is known. These authors have argued that the signature of conflict monitoring on correct trials is reflected in the N2 ERP component of the target epoch. In contrast, the conflict monitoring associated with errors takes place after the response and, thus, is seen in the response epoch in the well-known error-related negativity (ERN; see, e.g., Yeung, Botvinick, \& Cohen, 2004; Yeung $\&$ Cohen, 2006). These elaborations of the theory were made to address the usual lack of post response conflictrelated ERP effects (but see Bartholow et al., 2005; Forster $\&$ Pavone, 2008). The conflict-monitoring theory seems to make the prediction that the conflict adaptation contrast also will not be significant during the response epoch. Nonetheless, there are some indications that task switching creates a special case in this regard. Specifically, Hsieh and Liu (2009) examined the role of stimulus bivalencenamely, whether the target stimulus afforded the two tasks that were involved (bivalent) or afforded only the relevant task (univalent). These authors found a modulation of the P3b component of the ERP on the following trial, suggesting that the conflict between tasks that takes place with bivalent stimuli had an influence beyond the target epoch of the trial in which this conflict took place.

Cue epoch The CRS effect is arguably related to inhibition (and related difficulty in reactivation) of abstract task rule representations. It thus should be observed in ERPs during the epoch in which the abstract task identity information is retrieved, thus indicating inhibition-related retrieval difficulty. Jamadar Hughes, Fulham, Michie, and Karayanidis (2010) who measured both ERP and fMRI BOLD signals, found that, relative to uninformative cues (fixation point), informative task cues (carrying task identity information and, thus, involving task identity retrieval) generated a positive deflection in the cue-locked ERP. This positivity was observed in the 300 to $400-\mathrm{ms}$ cue-locked time window, well before switch-related positivity was observed (450 to 550-ms time window). Visual inspection of Fig. 2 in their article suggests that the informative cue positivity actually started to appear about $250 \mathrm{~ms}$ after cue presentation. This early positivity showed positive correlations with the equivalent contrast on the FMRI BOLD activations observed in the right dorsal prefrontal cortex. Because the CRS effect presumably reflects difficulties in retrieving a task rule that was conflict generating before hand, Jamadar et al.'s results lead us to predict a CRS positivity in a 
A

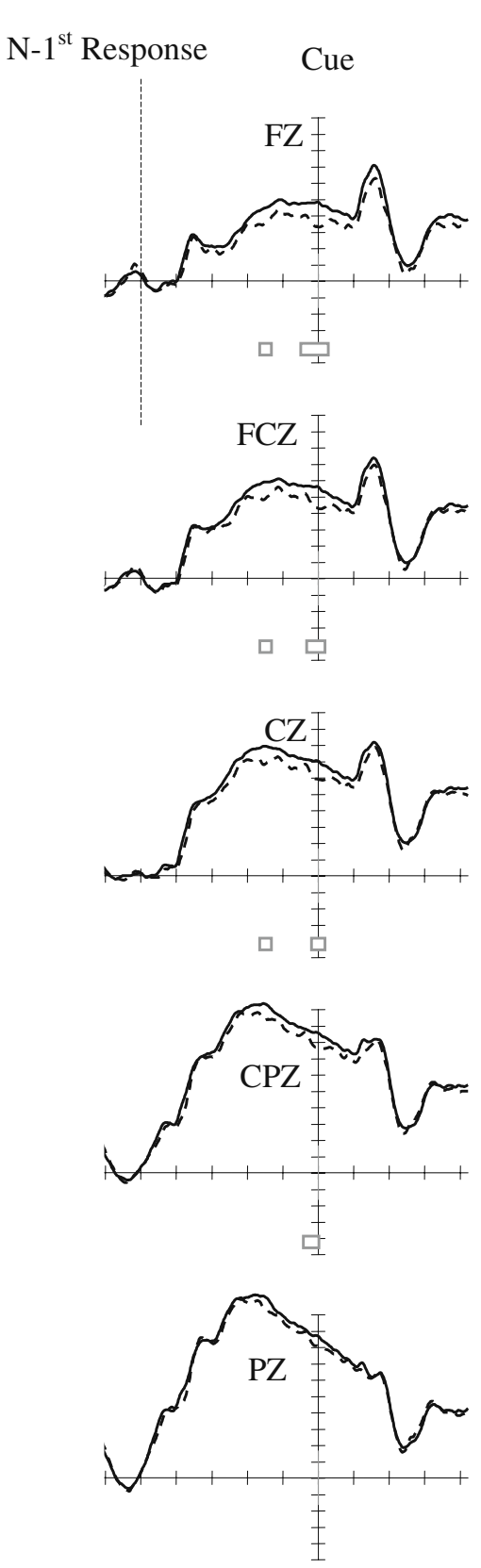

Fig. $2 n-1$ response epoch ERPs: Conflict adaptation contrast. a Left column: $n-1$ response-locked ERPs at five midline electrode sites. Time periods of consecutive significance are shown by the bars below the waveforms. Open bars indicate regions of significantly greater negativity for $\mathrm{CRS}+$ and post-incongruent trials, as compared

similar time window and, possibly, generated in the dorsal prefrontal cortex.

For completeness sake, we additionally review other studies, but none of them examined CRS. Thus, the reader who wishes to focus just on the main issue may skip this review and move directly to The Present Experiment section.

\section{B}
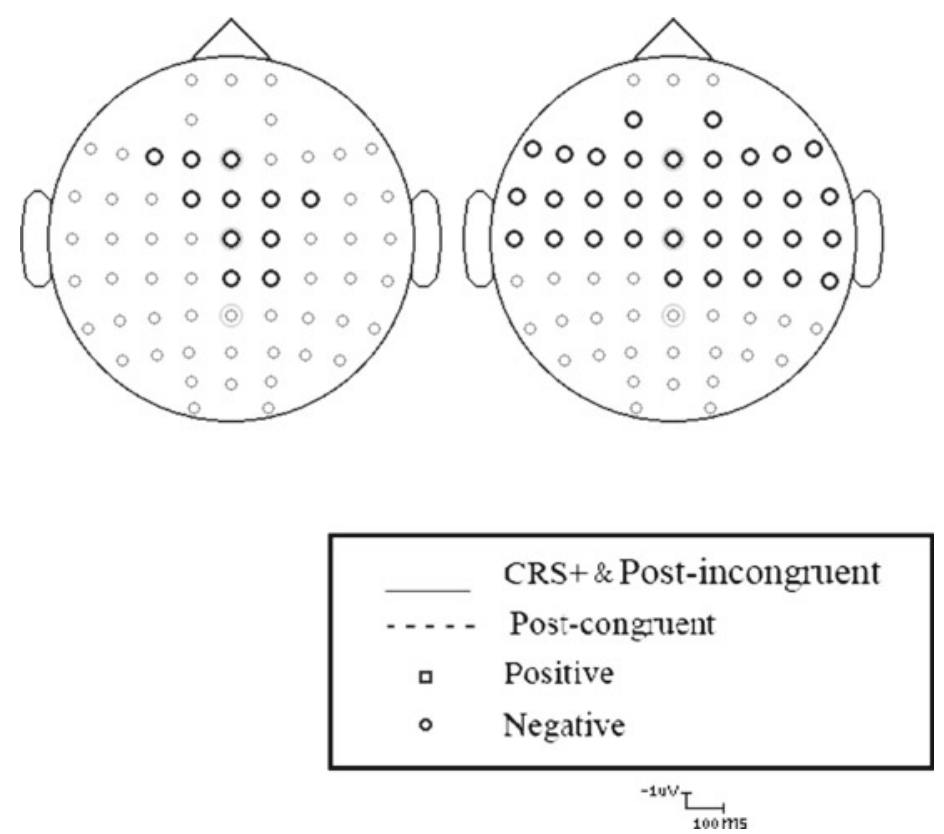

with post-congruent trials. b Scalp maps showing the sites of significant contrast in the epochs-of-interest. Dark circles indicate significantly greater negativity for CRS + and post-congruent as compared with post-congruent

A number of studies have focused on cue processing (see Karayanidis et al., 2010, for a review). Many of these studies have reported that in comparison with task repeat trials, there was a switch-related central-posterior positivity occurring about $400 \mathrm{~ms}$ after the cue (Hsieh, 2002; Karayanidis, Coltheart, Michie, \& Murphy, 2003; Miniussi, Marzi, \& Nobre, 2005; Moulden et al., 1998; Rushworth, 
Passingham, \& Nobre, 2002) or sometimes earlier (Slagter, Kok, Mol, Talsma, \& Kenemans, 2005). This positivity was interpreted by some authors as P3b modulation (Barceló, Periáñez, \& Knight, 2002; Kieffaber \& Hetrick, 2005; Nicholson, Karayanidis, Poboka, Heathcote, \& Michie, 2005; Tieges, Snel, Kok, Plat, \& Ridderinkhof, 2007) and by others as a reduction in CNV (Hsieh \& Chen, 2006; Lorist et al., 2000). The posterior positivity is sometimes preceded by an early (100 to $300-\mathrm{ms}$ ) or moderately early (300 to 500-ms) anterior positivity (Astle, Jackson, \& Swainson, 2008; Rushworth et al., 2002) or followed by a late (500 to 1,000-ms) anterior negativity (Astle et al., 2008; Lavric, Mizon, \& Monsell, 2008; Moulden et al., 1998). In other studies, the posterior positivity was preceded by an early anterior negativity (Poulsen, Luu, Davey, \& Tucker, 2005). The early anterior negativity coupled with later posterior positivity was also found when repeat trials taken from mixed-task blocks (including both switch and repeat) were compared with single-task (pure) blocks, suggesting that similar neural processes are engaged in task switch trials relative to task repeat trials, but also in task repeat trials relative to trials taken from pure blocks (Ruge, Stoet, \& Naumann, 2006).

In a recent review article, Karayanidis and colleagues (2010) further indicated that the cue-locked differential switch positivity (larger centro-parietal positivity for switch) can be decomposed into a number of subcomponents associated with stimulus set preparation. These components are sensitive to shifting attention between stimulus features, stimulus sets and stimulus-response mapping, and are modulated by the degree to which the upcoming stimulus is prone to interference (e.g., Karayanidis et al., 2009; Nicholson, Karayanidis, Davies, \& Michie, 2006; Poulsen et al., 2005, cf. Jamadar et al., 2010). In addition, Karayanidis et al. (2010) pointed out another switchspecific preparatory component-that is, the late, slow frontal negativity (fronto-central pretarget negativity) - that is thought to be particularly associated with response set preparation processes (Astle et al., 2008; Jamadar et al., 2010; Mueller, Swainson, \& Jackson, 2007). West et al. (2010; see also Travers \& West, 2008) made a further distinction between four ERP components - namely, frontal P2 (180-200 ms), frontal positivity (375-425 ms), P3 (300-350 ms), and parietal slow wave (400-1,000 ms)related to task switching. Following previous works, these authors suggested that the frontal P2 reflects change detection in the anterior cingulate cortex. In addition, the parietal slow wave is sensitive to aspects of actual task preparation.

Although the aforementioned studies on task switching have provided different switch-related cue-locked ERP components, it is difficult to make direct inferences regarding which one of these components will be affected by CRS, since none of the previous studies involved this or an analogue comparison. Nevertheless, since the present study, differing from the aforementioned studies, involved only task switch, but not task repeat, trials, we might expect to at least observe these already-known switch-related components to appear in the cue epoch for all condition trials. Moreover, if, for example, the frontal P2 (or P3) is found to be increased on CRS+ and/or post-incongruent trials, it may suggest that more effort is demanded for the process of change detection (or cue encoding) in order to counteract the residual inhibition from previous trials. Under this scenario, we may further predict a correlation between the component modulation and the respective behavioral effect. The direction of this correlation should be the same as that in the ERP deflection; namely, the correlation should be positive in the cue epoch.

Target epoch Several studies have shown that task switching results in less positivity or greater negativity, as compared with task repetition (e.g., Karayanidis et al., 2003; Kieffaber \& Hetrick, 2005; Nicholson et al., 2005), and these effects tend to show up quite early. Of interest here is the suggestion that the modulation of the P3b ERP component (e.g., Barceló et al., 2002; Gehring, Bryck, Jonides, Albin, \& Badre, 2003; Hsieh \& Chen, 2006; Karayanidis et al., 2003; Kieffaber \& Hetrick, 2005; Wylie, Javitt, \& Foxe, 2003) represents the inhibition of the task set as a whole (Hsieh \& Liu, 2008, 2009). Among these studies is Hsieh and Liu's (2009) study that was described beforehand. We retrieved and reanalyzed Hsieh and Liu's (2009) data by further separating preceding bivalent trials into congruent and incongruent trials. We found no significant target-locked P3b modulations as a function of the congruency status of the preceding bivalent target. On the basis of these null results, we did not expect significant P3b modulations for the conflict adaptation contrast in the present study.

\section{The present experiment}

To reiterate, we addressed the core question: Does CRS influence task rules? Our behavioral paradigm resembles the paradigm used by Meiran et al. (2010), aside from a few notable changes necessary to accommodate our measurement of ERPs. Specifically, the large display used by Meiran et al. increased the likelihood of eye movements during the experiment. In an effort to discourage eye movements, we reduced the size of the display, which also required changing the nature of the objects. Thus, the facerelated tasks were replaced by a shape (dot vs. line) task and a color task (green vs. red); these were tasks that could be performed on relatively small objects (see Fig. 1). The 
two spatial tasks were up-down (as in Meiran et al.'s [2010] study) and in-out. In other respects, the paradigm was very similar to that used by Meiran et al. Specifically, all the trials involved task switching, but the nature of the tasks to which participants switched was pseudorandomly determined. Because the target stimuli could be classified according to all the four rules and because the same two keypress responses were used in all the tasks, rules could compete in the sense that an irrelevant rule could indicate the competing keypress. Because each trial involved one relevant (to-be-executed) rule, there were three irrelevant rules that were not to be executed. Since each one of the irrelevant rules could be a competing rule, there were different degrees of TRCE, ranging from zero competing rules to three competing rules. In a purely behavioral study involving 32 participants, we verified that the new version of the paradigm produces significant TRCE and CRS effects. This study is not reported here for brevity sake.

\section{Method}

\section{Participants}

Twenty-four participants (12 of them female; age range, 18-24 years; mean age, $20.5 \pm 1.77$; mean years of education, $15.0 \pm 1.40$ ) were recruited from National Chung Cheng University, Taiwan. All of the participants were right-handed, were free of neurological and psychological disorders, and had self-reported normal or correctedto-normal vision. Each participant completed an informed consent form and was paid NT \$500 (US \$15.50) for participating in the experiment.

\section{Behavioral paradigm}

\section{Stimuli and procedure}

The experiment was run on a Pentium 4 computer with a 17 -in. monitor. The software was programmed in E-Prime 1.0 (Schneider, Eschman, \& Zuccolotto, 2002). The stimuli consisted of a vertical array of four boxes, subtending a visual angle of $1.43^{\circ} \times 6.55^{\circ}$. The objects placed inside the boxes included a colored dot (diameter $\left.=0.47^{\circ}\right)$ and a colored line $\left(0.19^{\circ} \times 0.95^{\circ}\right)$. The task cues were transparent icons that were presented in the center of the vertical array of boxes. The experiment began with a verbal explanation and an illustration of the tasks and the stimuli. Afterward, the participants were required to execute four practice blocks in which the four tasks were added one by one until the participants had been exposed to all four tasks. Each experimental block consisted of 64 trials; so, without the practice blocks, there were 1,152 trials for each participant.
Participants rested for 2 min between blocks to stay alert. The total session took approximately $1 \mathrm{~h} 10 \mathrm{~min}$. The participants were asked to be as accurate and quick as possible.

A trial started with a response-cue interval of $500 \mathrm{~ms}$, during which a black screen was presented. This was followed by the task cue presentation for $600 \mathrm{~ms}$ and a blank screen for $100 \mathrm{~ms}$ and then by the target stimulus, which was kept on the screen until the response was given (see Fig. 1). A beep sound of $400 \mathrm{~Hz}$ was played after the response if an error was made. Participants indicated their response by pressing one of two keys on the keyboard, L (right) and A (left), according to the instructed category-toresponse mapping (e.g., "IF \{green\} THEN \{Press right\}") and according to the currently relevant task rule, color (green vs. red), shape (dot vs. line), up-down, and in-out (whether the shape occupied an inner or an outer box; see Fig. 1).

The response key arrangement was counterbalanced between participants. There were 16 counterbalancing conditions formed by all the combinations in which taskrelated categorizations (such as green and red for the color task) were mapped to the right-left keypresses. We fully counterbalanced key assignments for the first 16 participants and counterbalanced the response mappings of shape, in-out, and up-down, but not of color, for the additional 8 participants. ${ }^{2}$ The keypad was aligned with the center of the screen, and the participants were instructed to respond with their two index fingers.

\section{Design}

A preliminary set of analyses was conducted to detect whether a behavioral TRCE was occurring. Since there were four tasks, a given trial could involve zero to three competing rules representing four levels of incongruence (see Table 1). We reasoned that an increased number of competing rules would be associated with slower and less accurate responses. The core analysis involved the comparison of behavior and ERP amplitude in the three conditions, post-congruent, post-incongruent, and CRS+.

\section{EEG recording and EEG formation}

Participants were seated in a comfortable chair in a soundattenuated room during testing. Continuous EEG was recorded from 64 scalp electrodes using an electrode cap (Neuroscan Q-cap: AgCl-64 electrode cap; Neuroscan, Inc., El Paso, TX). Horizontal EOGs were recorded from

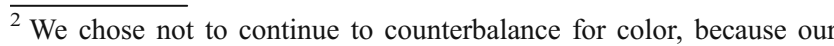
preliminary analyses indicated that there was only a 1-ms RT difference between the mapping conditions of this task.
} 
electrodes attached to the outer canthus of each eye. Meanwhile, vertical EOGs were recorded from two electrodes, one $2 \mathrm{~cm}$ below and one $2 \mathrm{~cm}$ above the left eye. EEGs were initially referenced online to the left mastoid and then referenced offline to the algebraic average of the left and right mastoids. Electrode impedances were maintained below $5 \mathrm{k} \Omega$. The EEG and EOG signals were amplified using Neuroscan Synamps 2 amplifiers (Neuroscan, Inc.). The signal was sampled at $500 \mathrm{~Hz}$ and filtered online using a high-pass filter of $0.1 \mathrm{~Hz}$ and a low-pass filter of $30 \mathrm{~Hz}$.

Ocular artifacts associated with blinks were corrected using the ocular artifact reduction algorithm provided by the Neuroscan software. Artifact rejection was subsequently performed via an algorithm (provided by the Neuroscan software) that rejects any epoch if the signal is below $-50 \mu \mathrm{V}$ or above $50 \mu \mathrm{V}$, if the drift of the EEG from baseline exceeds $50 \mu \mathrm{V}$ or if the A/D converters become saturated. The total rejection rate across different conditions (roughly equally distributed across conditions) averaged about $2 \%$.

Response-locked, cue-locked, and target-locked ERP epochs were created by extracting 1,022-ms epochs around the onset of the response, cue, or target, respectively, including a 100-ms preonset interval. The epoched segments were baseline corrected by taking the average between -100 and $0 \mathrm{~ms}$ of the response, cue, or target, respectively, for each electrode separately and subtracting that average from each sample point in the electrode. This baseline correction was carried out for each condition, separately.

\section{Data analysis for core comparisons}

In order to reduce the number of statistical comparisons and, thus, prevent alpha inflation, we started by comparing the two conditions that were predicted to generate the largest difference (CRS + and post-congruent). The two theoretically driven orthogonal planned contrasts, CRS (CRS+ vs. post-incongruent) and conflict adaptation (CRS+ and post-incongruent vs. post-congruent) were conducted only in the electrodes and time samples in which the preliminary contrast (CRS + vs. post-congruent) was significant. This approach is somewhat analogous to beginning with an omnibus two degrees of freedom analysis of variance before conducting planned comparisons. We decided not to employ it, because the rank order of the conditions was predicted (beginning with post-congruent and ending with CRS + ), a fact that permitted us to examine the more powerful one degree of freedom contrast. This analytic approach was adopted for both behavior (RT and error rate) and ERPs.

ERP The epoched ERP segments for correct trials were averaged according to the conditions of the CRS analysis reported above (including $\mathrm{CRS}+$, post-congruent, and postincongruent trials). The $\mathrm{CRS}+$ and post-congruent trial conditions were compared via paired two-sided $t$-tests at each time sample (i.e., every 2-ms time point). In order to reduce the number of statistical comparisons to prevent alpha inflation, we used the following procedure in each one of the sets of analyses, including response locked, cue locked, and target locked.

Determing epochs-of-interest First, we determined epochsof-interest by examining only five midline electrodes (see, e.g., Fig. 2). We wish to emphasize that we do not make the claim that the epochs of interest are ERP components. As we see them, they represent only time windows in which the three conditions significantly varied,

For each one of the five midline electrodes, we ran a series of two-sided $t$-tests comparing ERP amplitudes between the two extreme conditions: CRS+ and postcongruent. An epoch was defined as an epoch of interest if the CRS+ and post-congruent conditions showed a significant difference ( $p<.05$, two-sided) that persisted for more than nine consecutive time samples (this criterion was set on the basis of the simulations based on Guthrie and Buchwald's [1991] procedure to control for Type 1 error, assuming an autocorrelation of .90 for two-sided $t$ tests, unlike the simulations in the original article, which dealt with one-sided $t$-tests). We used two-sided $t$-tests and assumed a very high autocorrelation (resulting in a stricter significance criterion) in order to partly compensate for the fact that we examined five electrodes in order to determine the epochs-of-interest.

Examining the planned contrasts (CRS conflict adaptation) within the epochs-of-interest Once epochs-of-interest were identified, we examined whether the significant overall variability was caused by CRS or by conflict adaptation. To this end, we adjusted the required number of consecutive time samples showing a significant difference according to the Guthrie-Buchwald approach, this time taking into account the much smaller number of time samples within any given the epoch of interest. The planned contrasts were now examined on all the electrodes in which the CRS+ versus post-congruent contrast was significant, and not only on the midline electrodes, in order to determine their scalp distribution. This approach still runs the risk of alpha inflation due to the large number of electrodes being examined. To show that the effects were genuine, we capitalized on the fact that for yet unknown reasons, greater difficulty (e.g., switch vs. repeat) generates positivity during the cue epoch and negativity during the target epoch and response epoch (see Karayanidis et al., 2010, for a review). If the significant results were caused by alpha inflation alone, half of the significant deflections should 
have been positive and half negative, and the same holds for correlations with behavior. Thus, for each epoch-ofinterest, we compared the actual distribution of positive/ negative deflections/correlations with that expected 50:50 distribution, using chi-square tests.

Correlations with behavior The final set of analyses involved computing the Pearson correlation between the ERP amplitude effects and the corresponding behavioral effects. These correlations were computed only for electrodes in which the ERP effect met the aforementioned significance requirements and only for the epochs (embedded inside the epochs-of-interest) in which the given ERP effect was significant. We predicted that the direction of the correlations would be the same as the direction of the ERP deflection. Namely, the correlations were predicted to be negative during the response and the target epochs and were predicted to be positive during the cue epoch.

Our analytic approach takes care of the number of comparisons by restricting the initial decisions determining the epochs-of-interest to five midline electrodes, by examining them with a single comparison (CRS + vs. post-congruent) and by using the Guthrie-Buchwald criterion. At the same time, the approach ensures sufficient statistical power by using an initial comparison that was predicted to be most powerful and by adjusting the Guthrie-Buchwald criterion to the reduced number of time samples in the epochs-of-interest. Our analyses also permitted us to validate the statistical approach by examining the CRS contrast in the response-locked ERPs as explained already. The fact that we did not find any significant epochs for the CRS contrast in the aforementioned interval verifies, at least to some degree, that our procedure was sufficiently conservative. At the same time, the fact that we found significant epochs for both of these contrasts verifies that the procedure was not overly conservative.

SLORETA On the basis of the scalp-recorded electric potential distribution, the standardized low-resolution brain electromagnetic tomography (sLORETA) software (by Pascual-Marqui, 2002, publicly available free academic software at http://www.uzh.ch/keyinst/loreta.htm) was used to compute the cortical three-dimensional distribution of current density. sLORETA was conducted on individual ERPs for CRS+ and post-incongruent trials over the same epochs-of-interest as those used for the amplitude analyses (as described in the section above). The SLORETA/eLORETA software package was used to perform the statistical analyses. "Perform randomization SnPM, and compute bullet proof, corrected critical thresholds and p-values" under the "Statistics" subprogram of the "sLORETA/eLORETA" package was used, and the thresholds obtained by this procedure were used for declaring significant locations. A three-shell spherical head model registered to the Talairach human brain atlas was used (Talairach \& Tournoux, 1988).

\section{Results}

Behavioral results

The two trials following an error and the first two trials in each block were excluded from the analyses. This precaution was taken because errors in task switching are often associated with executing the wrong task (Hübner \& Druey, 2006; Meiran \& Daichman, 2005). Additionally, trials associated with an error or with an RT outside the 100 to 3,000-ms window were analyzed for accuracy, but not for RT. We conducted two separate sets of analyses. The analysis of TRCE was carried out on all the trials. We first examined whether rule incongruency resulted in response conflict. The mean correct RTs were 579, 598, 617, and $625 \mathrm{~ms}$ for zero to three incongruent rules, respectively, $F(3,69)=15.51, p<.0001$. The mean proportions of errors were $.016, .036, .067$, and .101 , respectively, $F(3,69)=32.99, p<.0001$. These results indicate that performance was adversely influenced by the presence of competing responses coming from irrelevant task rules, justifying the following core analyses of CRS.

In the core analysis, we included trials preceded by either fully congruent trials or trials involving only one competing rule. The mean RTs were 610,600 , and $592 \mathrm{~ms}$ for CRS+, post-incongruent, and post-congruent, respectively. We used logarithmically transformed RTs for the statistical comparison in an effort to reduce the influence of outliers and to increase the statistical power. In addition, we adopted a one-sided significance criterion in the analysis of behavior, given the fact that this was a replication of an earlier behavioral study. We began the analysis with the contrast comparing the two most extreme conditions, CRS + and post-congruent. This difference reached statistical significance, $t(23)=2.30, p<.02$, onesided. Given the fact that this difference was significant, we computed the two theoretically meaningful contrasts. The CRS contrast (CRS + vs. post-incongruent) was significant, $t(23)=1.75, p<.05$, one-sided. However, the conflict adaptation contrast (post-incongruent vs. post-congruent) was not significant, $t(23)=1.25, p=.11$, one-sided. The mean error rate in the three conditions was $.058, .053$, and .052 , respectively. Although the raw trend indicated an effect of CRS on errors, none of the pair wise differences in error rates approached significance. These results indicate that CRS, but not conflict adaptation, affected participants' behavior. Note that the size of the behavioral effects is rather modest, as compared with the usual effects of around $30 \mathrm{~ms}$, as seen in numerous unpublished 
behavioral experiments in our lab, including an experiment with exactly the same behavioral paradigm as that used here.

An additional analysis was carried out to validate the inhibitory interpretation of the CRS effect (we wish to thank Todd Braver for pointing out this prediction to us). The logic behind this analysis was that if a rule was suppressed on trial $n-1$ and the same rule competed on trial $n$, the fact that the competing rule was already (partially) suppressed should have facilitated performance. We therefore classified the trials as involving a repetition of the competing rule or not. We also focused just on trials with one competing rule on both trial $n-1$ and trial $n$. The reason for this choice was to avoid examining complex interactions between various competing rules, especially given the fact that we did not have clear predictions regarding such interactions. The results indicated quicker responses for repeated competing rules $(573 \mathrm{~ms})$ than for nonrepeating competing rules (605 $\mathrm{ms})$, as was predicted, $t(23)=1.75, p<.05$, one-sided. The trend for errors was the same ( $3.6 \%$ vs. $4.5 \%$, respectively), but it did not approach significance $(p=.33)$.

\section{Electrophysiological results}

As in the core behavioral analyses, we analyzed only trials following a congruent trial or following trials with a single competing rule. The results are presented in Figs. 2, 3, 4 and 5. We present only the results in which the planned theoretically driven contrasts were significant, and we do not report the results of the preliminary contrast between $\mathrm{CRS}+$ and post-congruent, which was carried out only to determine the epochs-of-interest.

\section{Response-locked ERPs}

In these analyses, we focused on the epoch between the $n-1$ response to the $n$th cue.

The conflict adaptation contrast indicated a significant negative deflection in two time windows (see Fig. 2). In the 336 to 366-ms time window, the effect showed up in 11 fronto-parietal midline electrodes $(p<.001$, for the deviation from 50:50 positive/negative deflections expected by chance). In the 470 to $528-\mathrm{ms}$ time window, the negative deflection was observed in 34 electrodes $(p<.0001)$ distributed over the entire prefrontal cortex. The ERP effect was uncorrelated with the respective behavioral effect, though.

The CRS contrast did not yield any significant results. This fact provides validation to our statistical approach, since the CRS + and post-incongruent conditions are not differentiated logically before the $n$th cue is known. Thus, the lack of significant effects shows that our procedure was sufficiently conservative to prevent noise from causing significant effects.

\section{Cue-locked ERPS}

In these analyses, we focused on the cue-target interval. First, disregarding any contrast, the cue-locked ERPs indicated an anterior N2, a central P3, and a slow wave, as would be expected for switch trials (all the trials in this experiment were switch trials). The conflict adaptation contrast yielded significant results in five time windows (see Fig. 3). In all of them, the deflection was positive. The first time window was very early and brief (128-138 ms) and was shown only in 3 electrodes. Nonetheless, the fact that all these deflections were positive could still have been caused by chance alone $(p=.08$, for the deviation from 50:50). Together with the fact that it showed up only in three nonadjacent electrodes lead us to question its reliability. The next time window was the 202 to $218-\mathrm{ms}$ time window, in which the effect showed up in 21 electrodes over the entire posterior frontal cortex and above the right hemisphere $(p<.0001$, for the deviation from 50:50). The fact that the correlation between the ERP effect and the RT effect was significant in only 1 out of 22 electrodes leads us to suspect the reliability of this correlation, given that 1 out of 20 correlations should be significant by chance. In the 256 to $306-\mathrm{ms}$ time window, the effect was seen in 34 electrodes distributed over the entire central scalp $(p<.0001$, for the deviation from 50:50), but the correlations between the ERP effect and the RT effect were significant only in the right-sided electrodes. The fact that all the 12 significant correlations were positive (as was predicted) has a $p<.001$ for the deviation from the expected 50:50 positive/negative trend. In the 402 to 414-ms time window, the effect was seen in 14 electrodes $(p<.0005$, for the deviation from 50:50) over the left anterior cortex but was uncorrelated with the RT effect, and in the 560 to 586-ms time window, it was shown in 9 electrodes $(p<.005$, for the deviation from 50:50) distributed over the right anterior cortex, again without any significant correlations with the respective behavioral effect.

The CRS contrast showed significant positive deflections in two time windows (see Fig. 4). In the 248 to 262-ms time window, the effect was seen in most of the electrodes $(22 ; p<.0001$, for the deviation from 50:50) placed over the right hemisphere, with the exception of the very anterior and very right-lateralized electrodes. In none of these electrodes was there a significant correlation between the ERP effect and the behavioral effect. In the 274 to $298-\mathrm{ms}$ time window, the effect was seen in five electrodes $(p<.05$, for the deviation from 50:50) around the central midline $(\mathrm{Cz})$ region, and in two of them, there was a significant positive correlation (as was predicted) with the respective RT effect. This rate ( 2 out of 5 ) is well above that expected by chance ( 1 out of 20) (see Fig. 4 for the scatter plots of these correlations). 

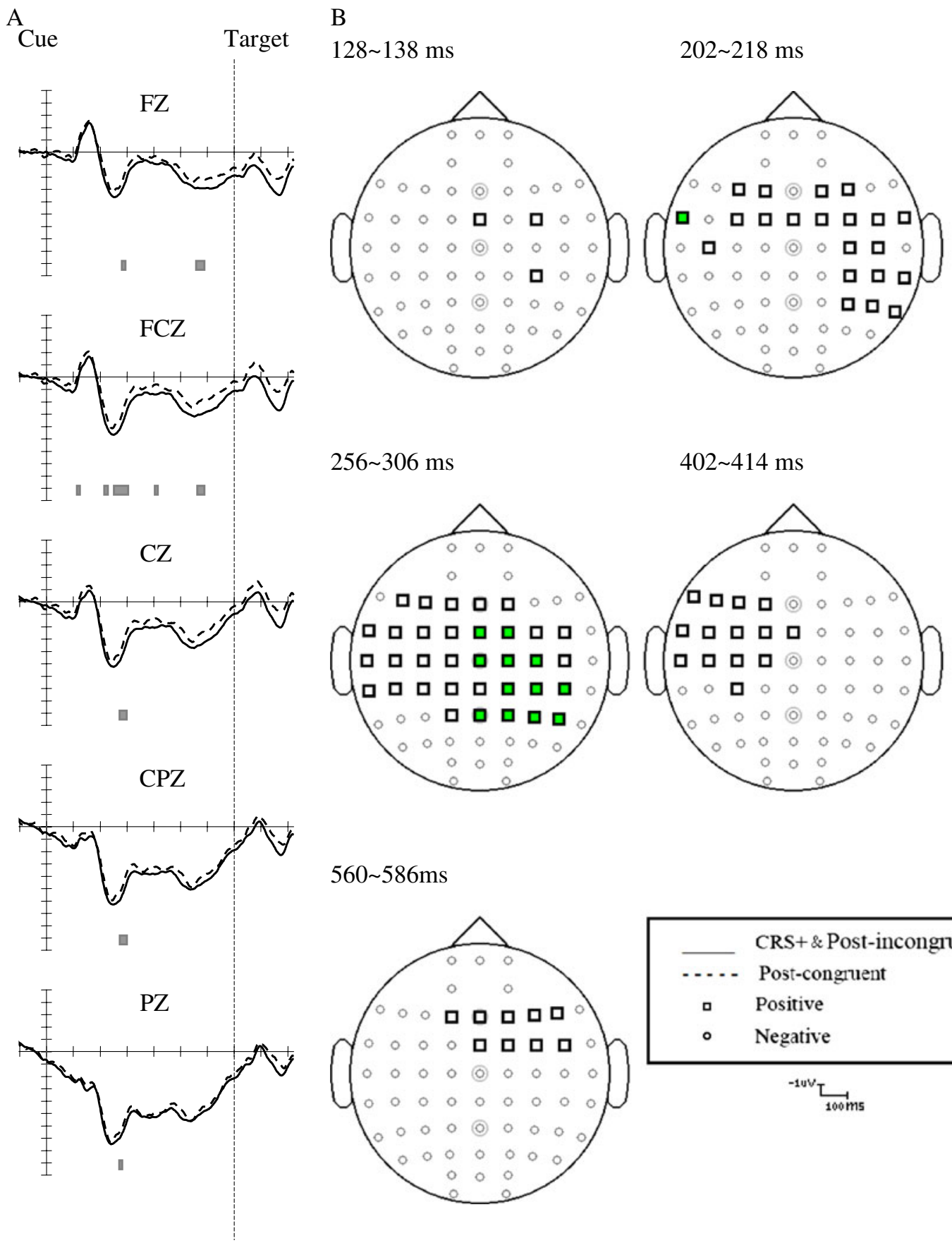

$560 \sim 586 \mathrm{~ms}$

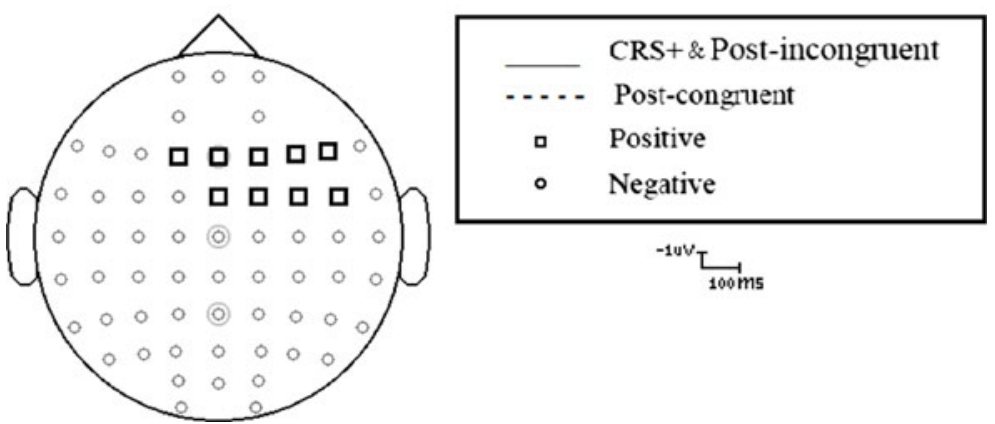

Fig. $3 N$ th cue epoch ERPs: Conflict adaptation contrast. a Left column: $n$th cue- locked ERPs at five midline electrode sites. Time periods of consecutive significance are shown by the bars below the waveforms. Filled gray bars indicate regions of significantly greater positivity for $\mathrm{CRS}+$ and post-incongruent trials. as compared with

\section{Target-locked ERPs}

In these analyses, we focus on the epoch following the presentation of the imperative stimulus, the target. The conflict adaptation contrast was not significant in this epoch. However, the CRS contrast indicated significant post-congruent trials. b Scalp maps showing the sites of significant contrast in the epochs-of-interest. Dark squares indicate significantly greater positivity for CRS+ and post-congruent, as compared with post-congruent. Green marks indicate a positive correlation with the respective RT effect

negative deflections in the ERP in three time windows (see Fig. 5). In the 348 to $370-\mathrm{ms}$ time window, the effect was seen in 5 electrodes $(p<.05$, for the deviation from 50:50) distributed over the right parietal cortex and in 4 out of 5 electrodes in which the effect was significant, it correlated negatively with the respective RT effect. In the 
A

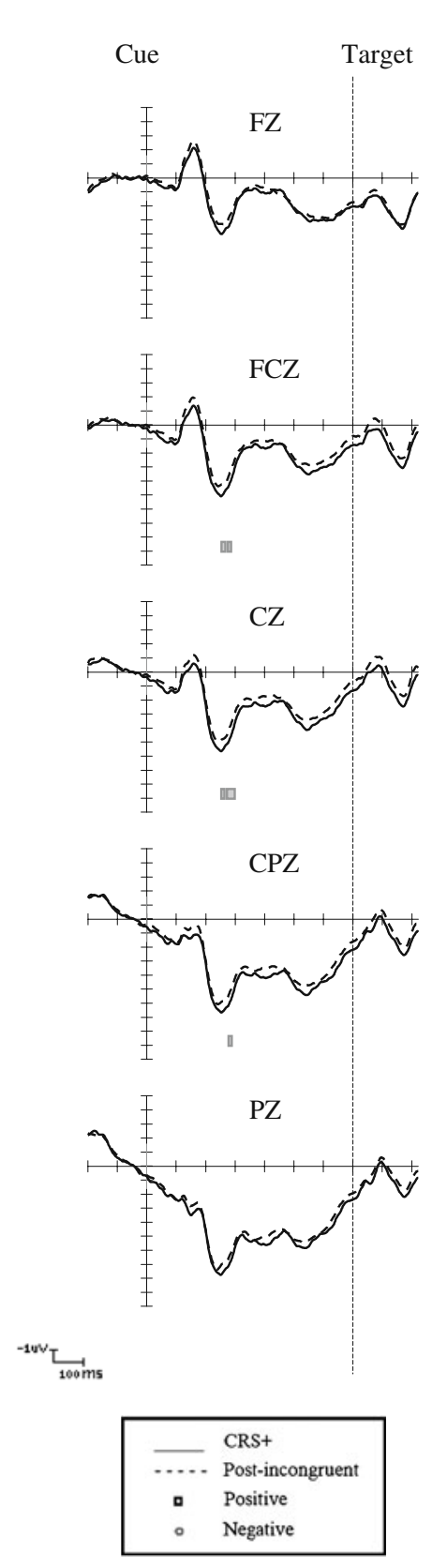
248 262ms
274 298ms

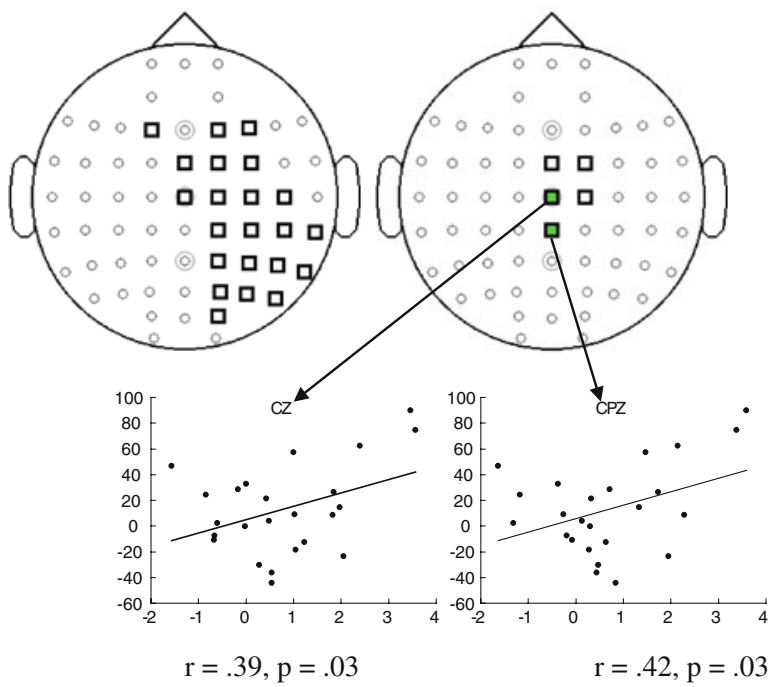

$\mathrm{C}$

a. $248 \sim 262 \mathrm{~ms}$

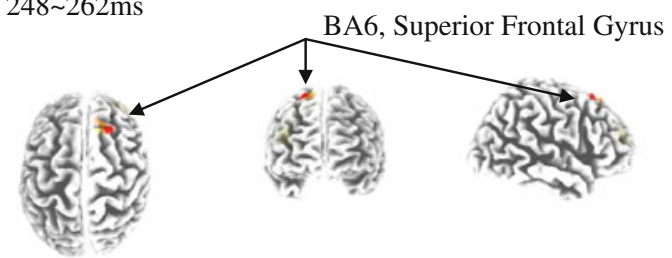

Axial View Frontal View Right View

b. 274 298ms BA6, Superior Frontal Gyrus

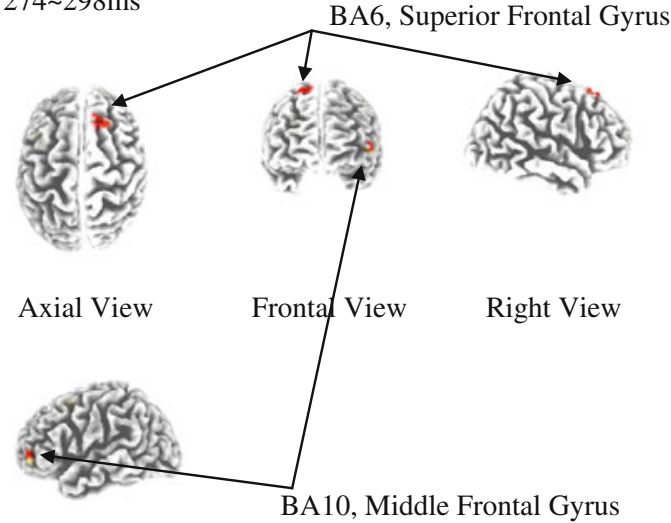

Left View
Fig. $4 N$ th cue epoch ERPs: CRS contrast. a Left column: $N$ th cuelocked ERPs at five midline electrode sites. Time periods of consecutive significance are shown by the bars below the waveforms. Filled gray bars indicate regions of significantly greater positivity for CRS + trials, as compared with post-incongruent trials. b Scalp maps showing the sites of significant contrast in the epochs-of-interest. Dark squares indicate significantly greater positivity for CRS + trials, as

460 to 486-ms time window, the effect was seen in 17 electrodes $(p<.0001$, for the deviation from 50:50) over the very posterior cortex but was uncorrelated with the RT effect. Finally, in the 534 to $570-\mathrm{ms}$ time window, the compared with post-incongruent trials. Green marks indicate a positive correlation with the respective RT effect; In the scatterplots of the two significant correlations, the abscissa is the CRS effect in ERP (in microvolts), and the ordinate is the CRS effect in reaction time (in milliseconds). c sLORETA source analysis results for the significant time windows (yellow $=p<.05$; orange $=p<.01$ )

effect was seen in 22 electrodes $(p<.0001$, for the deviation from 50:50) over the centro-parietal cortex, again without any significant correlations with the respective RT effect. 
Fig. $5 N$ th target epoch ERPs: CRS contrast. a Left column: $N$ th target-locked ERPs at five midline electrode sites. Time periods of consecutive significance are shown by the bars below the waveforms. Open bars indicate regions of significantly greater negativity (less positivity) for CRS + trials, as compared with postincongruent trials. b Scalp maps showing the sites of significant contrast in the epochs-of-interest. Dark circles indicate significantly greater negativity for CRS+ and post-congruent, as compared with post-congruent. Red marks indicate a negative correlation with the respective RT effect. c sLORETA source analysis results for the significant time windows (yellow $=$ $p<.05$; orange $=p<.01)$
A
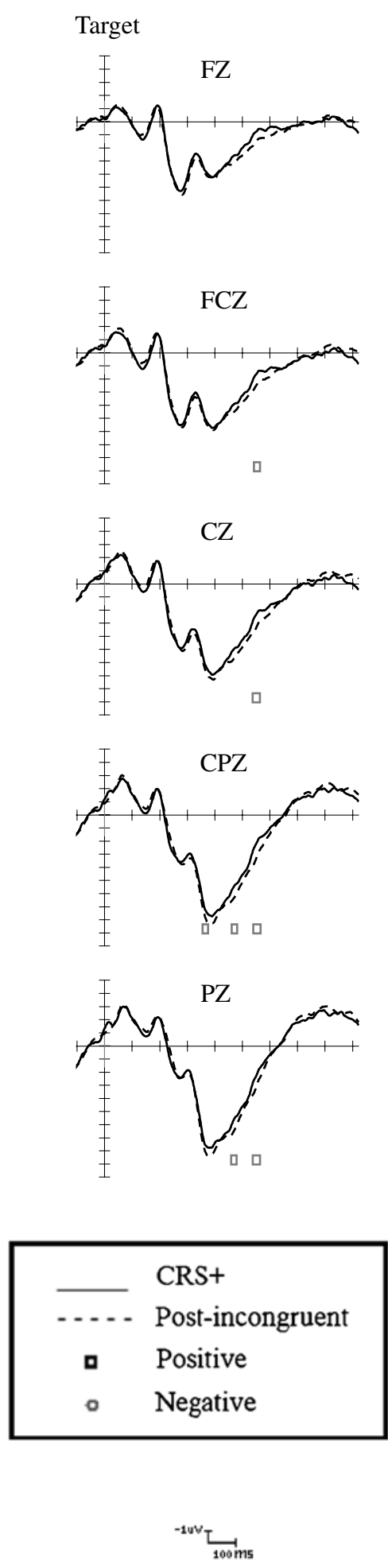

B

348 370 ms 460 486 ms
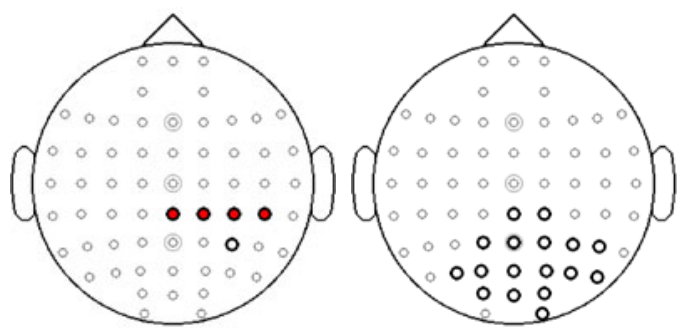

534 570 ms

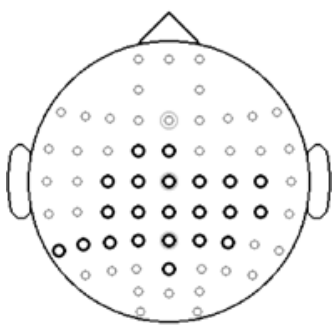

C

a. $348 \sim 370 \mathrm{~ms}$

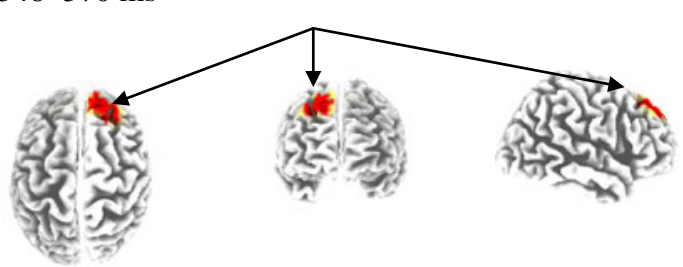

Axial View $\quad$ Front View Right View

b. $460 \sim 486 \mathrm{~ms}$

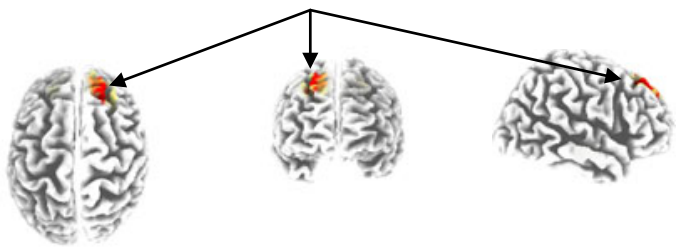

Axial View $\quad$ Front View Right View

c. 534 570ms

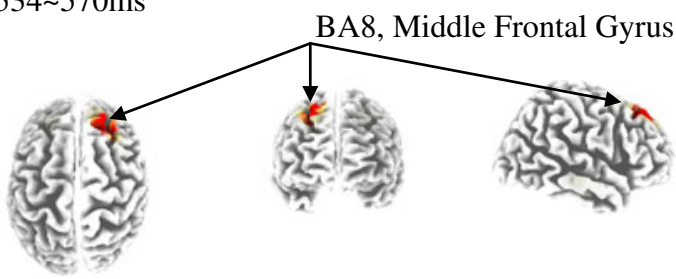

Axial View

Front View

Right View

\section{sLORETA}

In order to examine the neural generators for the CRS effect, we employed sLORETA (Pascual-Marqui, 2002). We ran the analysis separately for each time window in which the contrast was significant. The results are presented in Figs. 4 and 5. In all the five time windows (including those found in the cue epoch and target epoch), the sLORETA analyses indicated a significant source in the right dorsal prefrontal cortex, including Brodman areas 6, 8, 9, 10, and 
46. In one of the five analyses, there was an additional source in the left prefrontal cortex (Brodman areas 6, 10).

The post conflict adaptation contrast (not shown) was not associated with any significant source during the response epoch. In the cue epoch, there was a significant source in the medial wall of the right superior frontal gyrus and only in the 560 to $586-\mathrm{ms}$ time window, which is only one out of seven time windows (including those in the response epoch and the cue epoch) in which we observed a significant effect in the ERP amplitude.

\section{Discussion}

In this experiment, we addressed the question: Does CRS influence task rules (as opposed to more concrete representations involving stimuli and responses)? We reasoned that if the CRS effect is seen during the cue epoch, this would provide an affirmative answer to our question. The results indicate that this was indeed the case. Specifically, we identified two time windows in which the CRS+ condition generated a positive deflection in the ERP, relative to post-incongruent. Because, during the cue epoch, the identity of target is yet unknown and the same is true for the response, the effect cannot be explained as reflecting the inhibition of stimulus-related information (Tipper, 2001) or of responses and, thus, must be attributed to an influence of the task as a whole. Interestingly, our source localization results imply a brain region and epoch very similar to those identified by Jamadar et al. (2010) as being related to the retrieval of task identity information, as revealed in the comparison of informative and uninformative cues. Importantly, while task identity information (such as "the shape task") may be tied to the entire stimulus set and the entire response set (either "dot, line" or "right key, left key"), it is neither tied to a specific stimulus within the set nor tied to a specific response within the set. Thus, these results further support the conclusion that CRS operates at the level of the task set as a whole. The CRS contrast has yielded additional effects during the target epoch, but these effects are more difficult to interpret because they could indicate the inhibition of stimulus-related information, response inhibition, or the inhibition of particular categoryto-response associations. Of interest is the fact that the time windows above, as well as the scalp distribution of these target-locked effects, suggest that we observed a modulation of the $\mathrm{P} 3 \mathrm{~b}$, which was previously interpreted as reflecting the inhibition of the task set as a whole (Hsieh \& Liu, 2008, 2009).

We also computed correlations between the ERP effects and the respective RT effects. Correlations were found for the effects observed in the cue epoch and the target epoch, and not in the $n-1$ response epoch. This makes sense given the fact that RT is measured in trial $n$ after the stimuli for this trial (the cue and the target) have been provided. The direction of the correlations accords with the yet unexplained fact that, during the cue epoch, the more difficult condition (switch) is associated with positivity, while in the target epoch it is associated with negativity (e.g., Karayanidis et al., 2010). Specifically, participants for whom the more difficult condition (e.g., CRS+) was especially difficult (leading to prolonged RT) showed greater positivity during the cue epoch and greater negativity during the target epoch.

At the conceptual level, the CRS effect resembles post conflict adaptation. In both cases, the detection of conflict leads to subsequent behavioral and brain responses. The main difference between the two mechanisms is their degree of specificity. Conflict adaptation can be conceived of as a relatively crude means for dealing with the conflict. In contrast, the CRS represents smart inhibition that can be finely targeted at the competing rule. The fact that the post conflict adaptation contrast and the CRS contrast yielded different results further supports their conceptual distinction. We wish to acknowledge again the fact that while our study design was optimally suited for studying CRS effects, it was less optimally suited for studying conflict adaptation. For that reason, the conflict adaptation results are secondary in nature. In any event, it is difficult to see how conflict adaption could have predicted an influence on a specific task rule without a mechanism detecting the competing rule, which is what CRS is all about.

Before continuing the discussion, we would like to rule out an alternative explanation based on a potential confound with activity in the preceding trial. This confound exists in our paradigm because we used a fixed intertrial interval. Thus, cue-and target-locked activity that we attributed to trial $n$ might have been generated on trial $n-1$. Nonetheless, the conditions that were compared in the core CRS contrast were equal in terms of the conflict on trial $n-1$ and differed with respect to the relationship between trials $n-1$ and $n$, a fact that rules out the alternative account, at least for this comparison. The lack of a significant CRS effect during the target epoch further supports these conclusions.

Another potential confound is related to the fact that the more demanding conditions (as seen in behavior) produced negativity in the response epoch and positivity in the cue epoch. Thus, one could argue that the positivity in the cue epoch is simply due to a baseline shift. Namely, the baseline used to compute the cue epoch effects had a negative shift in the more demanding conditions. We argue that this alternative explanation is invalid for two main reasons. First, it predicts that the CRS effect would be absent in the cue epoch because it was absent in the response epoch. Second, the alternative explanation wrongly 
makes the prediction that the cue effects would be found in the time windows closest to the cue (in which the influence of the response epoch is largest), whereas we found that the effects were in fact delayed.

In our analyses, we also examined conflict adaptation effects and found such effects both in the response-to-cue epoch and in the cue epoch. We will focus here on the response epoch results because of the potential confound mentioned in the preceding paragraph. These results are intriguing, since the literature on conflict adaption suggests that in the case of correct responses (those which we have analyzed), the conflict is detected before the response is made and is, therefore,usually seen in the epoch preceding the response, in the N2 component, while post response effects are reflected in the ERN (Yeung et al., 2004). The effects that we observed took place in time windows that were quite remote from where the ERN is usually found. Hsieh and Liu (2009) also found modulations of the P3b on the trial following a bivalent trial (in which conflict between tasks takes place), suggesting that the influence of at least some conflicts may be relatively protracted.

The present results are relevant to wider issues discussed in the literature regarding task switching and cognitive control. Specifically, they add an important piece of evidence that the processing of task cues in taskswitching is sensitive to control demands and, therefore, involves cognitive control. This evidence is especially needed due to some influential theorizing that argues otherwise. Specifically, Logan and Bundesen (2003), as well as Schneider and Logan (2005), have argued that cue processing involves only perception and the immediate sensory-perceptual memory (of the previous cue). These authors argued that participants encode the task cue and the target stimulus and then react to the cue-target compound, effectively avoiding any task switching. These claims are incompatible with the evidence that cue processing is sensitive to CRS, as well as to other forms of task inhibition (Karayanidis et al., 2009; Nicholson et al., 2006; Sinai, Goffaux, \& Phillips, 2007). Therefore, the present results, like the recent results of Jamadar et al. (2010), are more in line with Mayr and Kliegl $(2000,2003)$, who argued that the cue epoch is devoted to the retrieval of task rules.

Before concluding, we wish to note that while the present results show that CRS operates at the level of the task rules, they cannot effectively distinguish between two explanations. One explanation is decaying inhibition, and it assumes that the inhibition that took place on trial $n-1$ did not completely decay and was thus seen on trial $n$. The alternative account is that the brain marks the competitor rule as to-be-suppressed for future encounters. According to this account, there was no inhibition during trial $n-1$.

In conclusion, the present results corroborate Meiran et al.'s (2010) results in showing evidence for a finely tuned form of inhibition. A major challenge in task switching is to maintain a delicate balance between high task readiness and being able to effectively control the resultant involuntary response activation. This activation is produced by currently irrelevant (but still highly accessible) task rules. Maintaining this delicate balance requires finely tuned forms of inhibition that can influence the rules that are generating incongruence without adversely influencing the other rules. As a result, inhibition blocks readiness to execute just one task, without compromising the readiness to execute other tasks.

Authors' note Nachshon Meiran, Department of Psychology and Zlotowsky Center for Neuroscience, Ben-Gurion University of the Negev, Beer-Sheva, Israel, 84105; Shulan Hsieh, Department of Psychology and Institute of Allied Health Sciences, National Cheng Kung University, Tainan, Taiwan, 701; Chi-Chih Chang, Department of Psychology, National Chung Cheng University, Chia-Yi, Taiwan, 621. Correspondence concerning this article should be sent to Nachshon Meiran, nmeiran@bgu.ac.il or Shulan Hsieh, psyhsl@mail.ncku.edu.tw.

The research was supported by a Bi-National Taiwan-Israel research grant to Shulan Hsieh and Nachshon Meiran.

We wish to thank Hanjung Liu for help in programming and collecting EEG data at the early stage of this research and American Journal Experts (www.journalexperts.com) for English proofreading.

\section{References}

Allport, D. A., Styles, E. A., \& Hsieh, S. (1994). Shifting intentional set: Exploring the dynamic control of tasks. In C. Umiltà \& M. Moscovitch (Eds.), Attention and performance XV: Conscious and nonconscious information processing (pp. 421-452). Cambridge: MIT Press.

Astle, D. E., Jackson, G. M., \& Swainson, R. (2008). Fractionating the cognitive control required to bring about a change in task: A dense-sensor event-related potential study. Journal of Cognitive Neuroscience, 20, 255-267.

Barceló, F. C. A., Periáñez, J. A., \& Knight, R. T. (2002). Think differently: A brain orienting response to task novelty. Neuroreport, 13, 1887-1892.

Bartholow, B. D., Pearson, M. A., Dickter, C., Sher, K. J., Fabiani, M., \& Gratton, G. (2005). Strategic control and medial frontal negativity: Beyond errors and response conflict. Psychophysiology, 42, 33-42.

Botvinick, M. M., Braver, T. S., Carter, C. S., Barch, D. M., \& Cohen, J. D. (2001). Evaluating the demand for control: Anterior cingulate cortex and crosstalk monitoring. Psychological Review, $108,624-652$.

Brown, J. W., Reynolds, J. R., \& Braver, T. S. (2007). A computational model of fractionated conflict-control mechanisms in task-switching. Cognitive Psychology, 55, 37-85.

Egner, T., \& Hirsch, J. (2005). Cognitive control mechanisms resolve conflict through cortical amplification of task-relevant information. Nature Neuroscience, 8, 1784-1790.

Forster, B., \& Pavone, E. F. (2008). Electrophysiological correlates of crossmodal visual distractor congruency effects: Evidence for response conflict. Cognitive, Affective \& Behavioral Neuroscience, 8, 65-73.

Forstmann, B. U., Brass, M., \& Koch, I. (2007). Methodological and empirical issues when dissociating cue-related from task-related 
processes in the explicit task-cuing procedure. Psychological Research, 71, 393-400.

Garcia-Garcia, M., Barceló, F., Clemente, I. C., \& Escera, C. (2010). The role of the dopamine transporter DAT1 genotype on the neural correlates of cognitive flexibility. The European Journal of Neuroscience, 31, 754-760.

Gehring, W. J., Bryck, R., Jonides, J., Albin, R., \& Badre, D. (2003). The mind's eye, looking inward? In search of executive control in internal attention switching. Psychophysiology, 40, 572-585.

Goschke, T. (2000). Intentional reconfiguration and involuntary persistence in task set switching. In S. Monsell \& J. Driver (Eds.), Control of cognitive processes: Attention and performance XVIII (pp. 331-355). Cambridge: MIT Press.

Guthrie, D., \& Buchwald, J. S. (1991). Significance testing of difference potentials. Psychophysiology, 28, 240-244.

Hsieh, S. (2002). Two component processes in switching attention: A study of event-related potentials. Perceptual and Motor Skills, 94, 1168-1176.

Hsieh, S., \& Chen, P. (2006). Task reconfiguration and carryover in task switching: An event-related potential study. Brain Research, 1084, 132-145.

Hsieh, S., \& Liu, H. (2008). Electrophysiological correlates of task conflicts in task-switching. Brain Research, 1203, 116-125.

Hsieh, S., \& Liu, H. (2009). Electrophysiological evidence of the adaptive task-set inhibition in task switching. Brain Research, $1255,122-131$.

Hübner, R., \& Druey, M. (2006). Response execution, selection, or activation: What is sufficient for response-related repetition effects under task shifting? Psychological Research, 70, 245261.

Jamadar, S., Hughes, M., Fulham, W. R., Michie, P. T., \& Karayanidis, F. (2010). The spatial and temporal dynamics of anticipatory preparation and response inhibition in taskswitching. Neuroimage, 51, 432-449.

Jost, K., Mayr, U., \& Rosler, F. (2008). Is task switching nothing but cue priming? Evidence from ERPs. Cognitive, Affective \& Behavioral Neuroscience, 8, 74-84.

Karayanidis, F., Coltheart, M., Michie, P. T., \& Murphy, K. (2003). Electrophysiological correlates of anticipatory and poststimulus components of task switching. Psychophysiology, 40, 329-348.

Karayanidis, F., Jamadar, S., Ruge, H., Phillips, N., Heathcote, A., \& Forstmann, B. U. (2010). Advance preparation in task-swtiching: Converging evidence from behavioral, model-based and brain activation approaches. Frontiers in Psychology, 1, 25. doi:10.3389/ fpsyg.2010.00025

Karayanidis, F., Mansfield, E. L., Galloway, K. L., Smith, J. L., Provost, A., \& Heathcote, A. (2009). Anticipatory reconfiguration elicited by fully and partially informative cues that validly predict a switch in task. Cognitive, Affective \& Behavioral Neuroscience, 9, 202-215.

Kieffaber, P. D., \& Hetrick, W. P. (2005). Event-related potential correlates of task switching and switch costs. Psychophysiology, $42,56-71$.

Kiesel, A., Steinhauser, M., Wendt, M., Falkenstein, M., Jost, K., Philipp, A. M., et al. (2010). Control and interference in task switching-A review. Psychological Bulletin, 136, 849-874.

Koch, I., Gade, M., Schuch, S., \& Philipp, A. M. (2010). The role of inhibition in task switching: A review. Psychonomic Bulletin \& Review, 17, 1-14.

Lavric, A., Mizon, G. A., \& Monsell, S. (2008). Neurophysiological signature of effective anticipatory task-set control: A taskswitching investigation. The European Journal of Neuroscience, 28, 1016-1029.

Logan, G. D., \& Bundesen, C. (2003). Clever homunculus: Is there an endogenous act of control in the explicit task-cuing procedure?
Journal of Experimental Psychology. Human Perception and Performance, 29, 575-599.

Lorist, M., Klein, M., Nieuwenhuis, S., De Jong, R., Mulder, G., \& Meijman, T. (2000). Mental fatigue and task control: Planning and preparation. Psychophysiology, 37, 614-625.

Mayr, U., \& Kliegl, R. (2000). Task-set switching and long-term memory retrieval. Journal of Experimental Psychology. Learning, Memory, and Cognition, 26, 1124-1140.

Mayr, U., \& Kliegl, R. (2003). Differential effects of cue changes and task changes on task-set selection costs. Journal of Experimental Psychology. Learning, Memory, and Cognition, 29, 362-372.

Meiran, N. (2010). Task switching: Mechanisms underlying rigid vs. flexible self control. In R. Hassin, K. Ochsner, \& Y. Trope (Eds.), Self control in society, mind and brain (pp. 202-220). New York: Oxford University Press.

Meiran, N., \& Daichman, A. (2005). Advance task preparation reduces task error rate in the cuing task-switching paradigm. Memory \& Cognition, 33, 1272-1288.

Meiran, N., Hsieh, S., \& Dimov, E. (2010). Resolving task rule incongruence during task switching by competitor rule suppression. Journal of Experimental Psychology. Learning, Memory, and Cognition, 36, 992-1002.

Meiran, N., \& Kessler, Y. (2008). The task rule congruency effect in task switching reflects activated long-term memory. Journal of Experimental Psychology. Human Perception and Performance, $34,137-157$.

Miniussi, C., Marzi, C. A., \& Nobre, A. C. (2005). Modulation of brain activity by selective task sets observed using event-related potentials. Neuropsychologia, 43, 1514-1528.

Monsell, S. (2003). Task switching. Trends in Cognitive Sciences, 7, 134-140.

Moulden, D. J. A., Picton, T. W., Meiran, N., Stuss, D. T., Riera, J. J., \& Valdes-Sosa, P. (1998). Event-related potentials when switching attention between task-sets. Brain and Cognition, 37, 186-190.

Mueller, S. C., Swainson, R., \& Jackson, G. M. (2007). Behavioural and neurophysiological correlates of bivalent and univalent responses during task switching. Brain Research, 1157, 56-65.

Nicholson, R., Karayanidis, F., Davies, A., \& Michie, P. T. (2006). Components of task-set reconfiguration: Differential effects of 'switch-to' and 'switch-away' cues. Brain Research, 1121, 160 176.

Nicholson, R., Karayanidis, F., Poboka, D., Heathcote, A., \& Michie, P. T. (2005). Electrophysiological correlates of anticipatory taskswitching processes. Psychophysiology, 42, 540-554.

Periáñez, J. A., \& Barceló, F. (2009). Updating sensory versus task representations during task-switching: Insights from cognitive brain potentials in humans. Neuropsychologia, 47, 1160-1172.

Pascual-Marqui, R. D. (2002). Standardized low-resolution brain electromagnetic tomography (sLORETA): Technical details. Methods and Findings in Experimental and Clinical Pharmacology, 24(Suppl. D), 5-12.

Poulsen, C., Luu, P., Davey, C., \& Tucker, D. M. (2005). Dynamics of task sets: Evidence from dense-array event-related potentials. Cognitive Brain Research, 24, 133-154.

Ruge, H., Stoet, G. T., \& Naumann, E. (2006). Attentional set mixing: Effects on target selection and selective response activation. Psychophysiology, 43, 413-421.

Rushworth, M. F. S., Passingham, R. E., \& Nobre, A. C. (2002). Components of switching intentional set. Journal of Cognitive Neuroscience, 14, 1139-1150.

Schneider, D. W., \& Logan, G. D. (2005). Modeling task switching without switching tasks: A short-term priming account of explicitly cued performance. Journal of Experimental Psychology. General, 134, 343-367. 
Schneider, W., Eschman, A., \& Zuccolotto, A. (2002). E-Prime user's guide. Pittsburgh: Psychology Software Tools.

Sinai, M., Goffaux, P., \& Phillips, N. A. (2007). Cue- versus responselocked processes in backward inhibition: Evidence from ERPs. Psychophysiology, 44, 596-609.

Slagter, H. A., Kok, A., Mol, N., Talsma, D., \& Kenemans, J. L. (2005). Generating spatial and nonspatial attentional control: An ERP study. Psychophysiology, 42, 428-439.

Talairach, J., \& Tournoux, P. (1988). Co-planar stereotaxic atlas of the human brain: 3-dimensional proportional system:An approach to cerebral imaging. New York: Thieme Medical.

Tieges, Z., Snel, J., Kok, A., Plat, N., \& Ridderinkhof, R. (2007). Effects of caffeine on anticipatory control processes: Evidence from a cued task-switch paradigm. Psychophysiology, 44, 561578.

Tipper, S. P. (2001). Does negative priming reflect inhibitory mechanisms? A review and integration of conflicting views. The Quarterly Journal of Experimental Psychology, 54A, 321343 .

Travers, S., \& West, R. (2008). Neural correlates of cue retrieval, task set configuration, and rule mapping in the explicit cue task switching paradigm. Psychophysiology, 45, 588-601.
Vandierendonck, A., Liefooghe, B., \& Verbruggen, F. (2010). Task switching: Interplay of reconfiguration and interference. Psychological Bulletin, 136, 601-626.

West, R., Langley, M. M., \& Bailey, K. (2010). Signaling a switch: Neural correlates of task switching guided by task cues and transition cues. Psychophysiology, 48, 612-623. doi:10.1111/ j.1469-8986.2010.01123.x.

Wylie, G. R., Javitt, D. C., \& Foxe, J. J. (2003). Task-switching: A high density electrical mapping study. Neuroimage, 20, 23222342.

Yeung, N., Botvinick, M. M., \& Cohen, J. D. (2004). The neural basis of error detection: Conflict monitoring and the error-related negativity. Psychological Review, 111, 931-959.

Yeung, N., \& Cohen, J. D. (2006). The impact of cognitive deficits on conflict monitoring: Predictable dissociations between the errorrelated negativity and N2. Psychological Science, 17, 164-171.

Yeung, N., \& Monsell, S. (2003). The effects of recent practice on task switching. Journal of Experimental Psychology. Human Perception and Performance, 29, 919-936.

Yeung, N., Nystrom, L. E., Aronson, J. A., \& Cohen, J. D. (2006) Between-task competition and cognitive control in task switching. The Journal of Neuroscience, 26, 1429-1438. 